\title{
基于能量算子的单信道重叠信号盲分离 方法
}

察权伟"，魏平，肖先赐

电子科技大学电子工程学院, 四川成都 610054

* E-mail: quanweicai@sina.com

收稿日期: 2007-02-24; 接受日期: 2007-07-17

摘要针对单个信道的多个时、频重叠信号分量的分离问题，提出一种能 量分离方法对各个信号分量进行瞬时频率和幅度估计，从而进行多个信号分 量的分离. 基于能量算子的计算简单, 以及很好的时域分辨率的特点, 该方 法可用于实时处理和实际工程应用. 仿真结果验证了该算法的有效性.

关键词

单信道 时频重叠 信号分量分离 能量算子 差分能量算子

单信道的多个信号分量时频重叠是非常普遍的问题，如：通信中的共道干扰、雷达系统中 的多个回波、生物医药工程中的胎儿和母亲的 ECG(electrocardiogram)信号重叠以及语音信号 处理中的鸡尾酒效应; 在电子侦察和对抗中它更是经常出现. 通常为了获取有用的信号分量, 需要进行信号分量分离, 因而单信道的多个时、频重叠信号分量的分离具有非常重要的实际意 义和应用价值.

对于多个时、频重叠信号分量的分离目前通常利用多个阵元的阵列接收或多信道接收, 然 后采用阵列信号处理方法或是盲信号处理方法进行多信号分量分离 ${ }^{[1]}$, 而对于单信道接收的 多个重叠信号分量的分离则无较好方法. 一般多是采用多项式拟和方法 ${ }^{[2-4]}$, 其计算较为复杂, 并且由于交叉项问题有可能造成错误估计，不利于实际的应用.

本文提出一种利用能量算子和差分能量算子的单信道多个时、频域重叠信号分量的分离 方法. 该方法通过理论分析得到了各个信号分量的瞬时幅度和瞬时频率估计关于信号能量函 数的解析表达式, 从而能够准确有效地估计各个信号分量的瞬时幅度和瞬时频率, 进而进行 信号分量的分离. 该算法计算简单、高效，并具有很好的时域分辨率以及瞬时自适应，而且不 需要知道信号的先验信息. 


\section{1 能量算子和高阶差分能量算子}

\section{1 单频信号的能量函数}

1990 年, Teager ${ }^{[5]}$ 提出了能量算子并利用其来进行非线性语音处理. 连续函数能量算子定 义为

$$
\Psi_{c}[x(t)] \triangleq[\dot{x}(t)]^{2}-x(t) \ddot{x}(t),
$$

其中 $\dot{x}(t)$ 和 $\ddot{x}(t)$ 分别为 $x(t)$ 对时间的一阶和二阶导数.

其后, Kaiser ${ }^{[6]}$ 系统地介绍了这些算子并利用它进行了单频信号的幅度和频率估计. 对于 单频信号 $x(t)=A \cos \left(\omega_{c} t+\theta\right)$, 它具有如下关系:

$$
\begin{aligned}
& \psi_{c}[x(t)] \triangleq A^{2} \omega_{c}^{2}, \\
& \psi_{c}[\dot{x}(t)] \triangleq A^{2} \omega_{c}^{4},
\end{aligned}
$$

则频率和幅度绝对值分别为

$$
\begin{aligned}
& \omega_{c}=\sqrt{\psi_{c}[\dot{x}(t)] / \psi_{c}[x(t)]}, \\
& |A|=\psi_{c}[x(t)] / \sqrt{\psi_{c}[\dot{x}(t)]} .
\end{aligned}
$$

对于离散信号 $x(n)=A \cos \left(\Omega_{c} n+\theta\right)$, 利用两个采样点后向差分 $[x(n)-x(n-1)] / T$ 代替连续信号 的导数 $\dot{x}(t)$. 不失一般性地假设 $T=1$, 则对应的离散信号的能量函数为

$$
\boldsymbol{\psi}_{d}[x(n)] \triangleq x^{2}(n)-x(n-1) x(n+1),
$$

可有

$$
\begin{gathered}
\boldsymbol{\psi}_{d}[x(n)] \triangleq A^{2} \sin ^{2}\left(\Omega_{c}\right), \\
\boldsymbol{\psi}_{d}[x(n)-x(n-1)] \triangleq 4 A^{2} \sin ^{2}\left(\Omega_{c} / 2\right) \sin ^{2}\left(\Omega_{c}\right),
\end{gathered}
$$

则数字频率和幅度绝对值分别为

$$
\begin{gathered}
\Omega_{c}=\arccos \left(1-\psi_{d}[x(n)-x(n-1)] / 2 \boldsymbol{\psi}_{d}[x(n)]\right), \\
|A|=\sqrt{\boldsymbol{\psi}_{d}[x(n)] /\left[1-\left(1-\boldsymbol{\psi}_{d}[x(n)-x(n-1)] / 2 \boldsymbol{\psi}_{d}[x(n)]\right)^{2}\right]} .
\end{gathered}
$$

\subsection{AM-FM 信号的能量函数}

1993 年, Maragos ${ }^{[7]}$ 近一步证明如果AM-FM信号的幅度、频率是慢变化时, 其能量函数和 单频信号具有相同的表达式形式. 即对于连续信号

$$
x(t)=a(t) \cos [\varphi(t)]=\left[1+k_{a} b(t)\right] \cos \left[\omega_{c} t+k_{f} \int_{0}^{t} q(\tau) \mathrm{d} \tau+\theta\right],
$$

这里假定 $0 \leqslant \omega_{m} \leqslant \omega_{c}, b(t)$ 和 $q(t)$ 为带限信号, $\omega_{a}$ 和 $\omega_{f}$ 分别为带限信号 $b(t)$ 和 $q(t)$ 的最高 频率, 且 $|q(t)| \leqslant 1,|b(t)| \leqslant 1$, 当 $\omega_{a}, \omega_{f}, k_{f} \ll \omega_{c}$ 时, 即信号幅度和频率变化为慢变化或与载频 比起来不大时, $x(t)$ 的能量函数为

$$
\psi[x(t)] \approx a^{2}(t) \omega_{i}^{2}(t)
$$




$$
\psi[\dot{x}(t)] \approx a^{2}(t) \omega_{i}^{4}(t) .
$$

可以看出此时 AM-FM信号与单频信号的能量函数表达式形式相同. 联合上面两式则瞬时频率 $\omega_{i}(t)$ 和瞬时幅度绝对值 $|a(t)|$ 估计与单频信号错误！未找到引用源。和错误！未找到引用源。 式一样.

如果 $b(t)$ 和 $q(t)$ 不是带限信号, 例如 $x(t)$ 为单频的指数幅度信号和 LFM 信号, 以及较大 的调制系数如 $k_{a}=0.5, k_{f} / \omega_{c}=0.2$, 这时利用(12)和(13)式的能量函数仍可获得较好的频率 和幅度绝对值估计.

通过采样, 连续信号 $x(t)$ 变为离散信号

$$
x(n)=a(n) \cos [\varphi(n)]=\left[1+K_{a} b(n)\right] \cos \left[\Omega_{c} n+K_{f} \int_{0}^{n} q(m) \mathrm{d} m+\theta\right],
$$

这里假定 $|q(n)| \leqslant 1, \quad K_{f} \leqslant \Omega_{c}, \quad a(n) \geqslant 0$, 则有

$$
\begin{gathered}
\psi_{d}[x(n)] \approx a^{2}(n) \sin ^{2}\left[\Omega_{i}(n)\right], \\
\psi_{d}[x(n)-x(n-1)] \approx 4 a^{2}(n) \sin ^{2}\left[\Omega_{i}(n) / 2\right] \sin ^{2}\left[\Omega_{i}(n)\right] .
\end{gathered}
$$

联合上面两式, 可得离散信号的瞬时频率 $\Omega_{i}(n)$ 和瞬时幅度绝对值 $|a(n)|$ 估计表达式与单频信 号的错误! 未找到引用源。和错误! 未找到引用源。式一样.

\section{2 多信号分量的能量分离方法}

利用能量算子虽能对单个 AM-FM 信号的瞬时频率和幅度绝对值进行很好的估计, 但如 果信号包含多个 AM-FM 信号分量, 由于能量算子的非线性, 将产生大量交叉项, 使其无法直 接进行信号分量的瞬时频率和幅度估计. 本文提出一种多信号分量的能量分离方法, 该方法 即使在多个信号分量时频域重叠的情形下也能获得很好的估计和分离效果.

\section{1 连续信号}

假设单个信道接收到的信号含有 $M$ 个信号分量.

$$
r(t)=\sum_{i=1}^{M} a_{i}(t) \cos \left[\varphi_{i}(t)\right]=\sum_{i=1}^{M}\left[1+k_{a_{i}} b_{i}(t)\right] \cos \left[\omega_{c_{i}} t+k_{f_{i}} \int_{0}^{t} q_{i}(\tau) \mathrm{d} \tau+\theta_{i}\right], \quad M \geqslant 2,
$$

其中 $a_{i}(t)$ 和 $\varphi_{i}(t)$ 是信号分量 $x_{i}(t)$ 的瞬时幅度和瞬时相位， $a_{i}(t)>0$ 信号分量为非常广泛和一 般的幅度和频率连续变化的窄带信号.

如果一个信号或信号分量的幅度、频率是慢变化的, 在每个时刻点或一段时间内可以把它 看成单频信号 ${ }^{[8]}$. 这样在短的时间 $\Delta t$ 内 $r(t)$ 可以表述为

$$
r(t)=\sum_{i=1}^{M} a_{i}\left(t_{0}\right) \cos \left[\omega_{i}\left(t_{0}\right) t+\phi_{i}\left(t_{0}\right)\right], \quad\left|t-t_{0}\right| \leqslant \Delta t,
$$

式中 $a_{i}\left(t_{0}\right)$ 和 $\omega_{i}\left(t_{0}\right)$ 及 $\varphi_{i}\left(t_{0}\right)$ 分别为信号分量 $x_{i}(t)$ 在 $t_{0}$ 时刻的瞬时幅度、瞬时频率和初相. 根据 单个正弦信号的微分关系, $r(t)$ 具有如下高阶微分方程: 


$$
\sum_{k=0}^{M} c_{k} r^{(2 k)}(t)=0, \quad c_{M}=1,\left|t-t_{0}\right| \leqslant \Delta t
$$

其中 $r^{(k)}(t)=\mathrm{d}^{n} r(t) / \mathrm{d} t^{n}$ 为 $r(t)$ 的高阶微分. 错误! 未找到引用源。式对 $r(t)$ 的高阶微分进行扩 展, 也同样成立, 故可以构建 $M$ 个方程

$$
\underbrace{\left[\begin{array}{cccc}
r^{(2 M-2)} & r^{(2 M-4)} & \cdots & r \\
r^{(2 M-1)} & r^{(2 M-3)} & \cdots & r^{(1)} \\
\vdots & \vdots & & \vdots \\
r^{(2 M+M-3)} & r^{(2 M+M-5)} & \cdots & r^{(M-1)}
\end{array}\right]}_{\boldsymbol{R}_{c}} \underbrace{\left[\begin{array}{c}
c_{M-1} \\
c_{M-2} \\
\vdots \\
c_{0}
\end{array}\right]}_{\boldsymbol{C}}=-\underbrace{\left[\begin{array}{c}
r^{(2 M)} \\
r^{(2 M+1)} \\
\vdots \\
r^{(2 M+M-1)}
\end{array}\right]}_{\boldsymbol{r}_{c}} .
$$

由于矩阵 $\boldsymbol{R}_{c}$ 是满秩的, 故有

$$
\boldsymbol{c}=-\boldsymbol{R}_{c}^{-1} \boldsymbol{r}_{c}
$$

定义 $k$ 阶差分能量算子 $\gamma_{\kappa}[r]$ 为 ${ }^{[9]}$

$$
\gamma_{\kappa}[r]=\dot{r} r^{(k-1)}-r r^{(k)},
$$

则可求得微分方程的系数 $\boldsymbol{c}=\left(c_{1}, \cdots, c_{M-1}\right)$ 为信号高阶差分能量函数的函数, 由此可求取微分 方程系数 $\boldsymbol{c}$ (见附录 $\mathrm{A}$ ).

$$
c_{i}=F_{1}\left(\gamma_{\boldsymbol{\kappa}}\left[r^{(\boldsymbol{m})}\right]\right), \quad i=0,1, \cdots, M-1 .
$$

理论分析微分方程系数 $c_{i}(i=0,1, \cdots, M-1)$ 可发现其为各个信号分量瞬时频率 $\omega_{i}\left(t_{0}\right)$, $(i=0,1, \cdots, M)$ 的基本对称多项式

$$
\begin{aligned}
& c_{0}=\omega_{1}^{2}\left(t_{0}\right) \cdot \omega_{2}^{2}\left(t_{0}\right) \cdots \omega_{M}^{2}\left(t_{0}\right), \\
& \cdots \\
& c_{M-2}=\sum_{i, j=1,(i<j)}^{M} \omega_{i}^{2}\left(t_{0}\right) \cdot \omega_{j}^{2}\left(t_{0}\right), \\
& c_{M-1}=\sum_{i=1}^{M} \omega_{i}^{2}\left(t_{0}\right),
\end{aligned}
$$

则瞬时频率 $\omega_{1}\left(t_{0}\right), \omega_{2}\left(t_{0}\right), \cdots, \omega_{M}\left(t_{0}\right)$ 就是方程

$$
\sum_{i=0}^{M}(-1)^{M-i} c_{M-i} \omega^{2 i}=0, \quad c_{M}=1
$$

的根. 联合错误! 未找到引用源。和错误! 未找到引用源。式, 可得各个信号分量的瞬时频率 $\omega_{i}\left(t_{0}\right)(i=0,1, \cdots, M)$ 也是信号高阶差分能量函数的函数, 即

$$
\omega_{i}\left(t_{0}\right)=F_{2}\left\{F_{1}\left(\gamma_{\kappa}\left[r^{(m)}\right]\right)\right\}, \quad i=0,1, \cdots, M,
$$

由此可由信号高阶差分能量函数估计出各个信号分量的瞬时频率. 但由 Abel 定理可知当信号 分量的个数大于 4 时, 错误! 未找到引用源。式没有一般的代数解法, 无法得到 
$\omega_{i}\left(t_{0}\right)(i=0,1, \cdots, M)$ 关于能量函数的解析表达式, 此时可以利用搜索求根的方法求得 $\omega_{i}\left(t_{0}\right)(i=0,1, \cdots, M)$.

估计出信号分量的瞬时频率后, 利用下式的瞬时幅度、瞬时频率、信号能量函数以及高阶 差分能量函数的关系, 进一步可以计算各个信号分量的瞬时幅度 $a_{1}\left(t_{0}\right), a_{2}\left(t_{0}\right), \cdots, a_{M}\left(t_{0}\right)$.

$$
\begin{aligned}
& \boldsymbol{\psi}_{c}[r] c_{0}-\boldsymbol{\psi}_{c}[\ddot{r}] c_{2}+\left(\boldsymbol{\psi}_{c}\left[r^{(3)}\right]-\boldsymbol{\gamma}_{4}[\ddot{r}]\right) c_{3}+\cdots+(-1)^{k+1}\left(\boldsymbol{\psi}_{c}\left[r^{(k)}\right]+\sum_{i=1}^{k-2}(-1)^{i} \gamma_{2 i+2}\left[r^{(k-i)}\right]\right) c_{k}+\cdots \\
& +(-1)^{M}\left(\boldsymbol{\psi}_{c}\left[r^{(M-1)}\right]+\sum_{i=1}^{(M-1)-2}(-1)^{i} \gamma_{2 i+2}\left[r^{((M-1)-i)}\right]\right) c_{M-1} \\
& +(-1)^{M+1}\left(\boldsymbol{\psi}_{c}\left[r^{(M)}\right]+\sum_{i=1}^{M-2}(-1)^{i} \gamma_{2 i+2}\left[r^{(M-i)}\right]\right) c_{M} \\
= & c_{0} \sum_{i}^{M} a_{i}^{2}\left(t_{0}\right) \omega_{i}^{2}\left(t_{0}\right)-2 c_{2} \sum_{i=1}^{M} a_{i}^{2}\left(t_{0}\right) \omega_{i}^{6}\left(t_{0}\right)+\cdots+(-1)^{k+1}(k-1) c_{k} \sum_{i=1}^{M} a_{i}^{2}\left(t_{0}\right) \omega_{i}^{2 k+2}\left(t_{0}\right)+\cdots \\
& -(M-2) c_{M-1} \sum_{i=1}^{M} a_{i}^{2}\left(t_{0}\right) \omega_{i}^{2(M-1)+2}\left(t_{0}\right)+(M-1) c_{M} \sum_{i=1}^{M} a_{i}^{2}\left(t_{0}\right) \omega_{i}^{2 M+2}\left(t_{0}\right),
\end{aligned}
$$

其中 $c_{M}=1$. 错误! 未找到引用源。式如同估计微分方程系数 $\boldsymbol{c}$ 一样, 同样也可以进行扩展构成 $M$ 个方程. 求解方程, 则可以得到 $a_{1}\left(t_{0}\right), a_{2}\left(t_{0}\right), \cdots, a_{M}\left(t_{0}\right)$ 的估计:

$$
a_{i}\left(t_{0}\right)=F_{3}\left(\psi_{c}\left[r^{(\boldsymbol{n})}\right], \gamma_{\kappa}\left[r^{(\boldsymbol{m})}\right], \boldsymbol{c}^{\prime}\right), \quad i=1, \cdots, M,
$$

式中

$$
\begin{gathered}
\boldsymbol{n}=(0,1, \cdots, 2 M-1), \boldsymbol{k}_{l}=(2 l+2), \boldsymbol{m}_{l}=(2,3, \cdots, 2 M-1-l), \quad l=1,2, \cdots, M-2, \\
\boldsymbol{c}^{\prime}=\left(c_{0}, c_{1}, c_{3}, c_{4}, \cdots, c_{M}\right) .
\end{gathered}
$$

对于其他时刻可像 $t_{0}$ 一样类推, 最后可以得到所有时刻信号分量的瞬时频率和瞬时幅度 的估计值, 然后重构各个信号分量, 即可实现对信号分量的分离.

\section{2 离散信号}

通常情形, 对于连续信号需要进行采样, 变成离散信号后进行处理, 此时 错误! 未找到引用源。式变为

$$
r(n)=\sum_{i=1}^{M} a_{i}(n) \cos \left[\Phi_{i}(n)\right]=\sum_{i=1}^{M}\left[1+k_{a} b(n)\right] \cos \left[\Omega_{c} n+K_{f} \int_{0}^{n} q(m) \mathrm{d} m+\theta\right], \quad M \geqslant 2,
$$

利用两个采样点后向差分 $[r(n)-r(n-1)] / T$ 代替连续信号的导数 $\dot{r}(t)$. 为了便于表示, 在后面 的公式中用 $r_{k}$ 表示 $r(n-k)$. 不失一般性地假设 $T=1$, 则错误 ! 未找到引用源。式的高阶微分 方程变为高阶差分方程

$$
d_{0} r_{M}+\sum_{k=1}^{M} d_{k}\left(r_{M-k}+r_{M+k}\right)=0, \quad d_{M}=1, \quad\left|n-n_{0}\right| \leqslant \Delta n .
$$

和连续信号一样可以构建 $M$ 个方程 


$$
\underbrace{\left[\begin{array}{cccc}
r_{1}+r_{2 M-1} & r_{2}+r_{2 M-2} & \cdots & r_{M}+r_{M} \\
r_{2}+r_{2 M} & r_{3}+r_{2 M-1} & \cdots & r_{M+1}+r_{M+1} \\
\vdots & \vdots & & \vdots \\
r_{M}+r_{3 M-2} & r_{M+1}+r_{3 M-3} & \cdots & r_{2 M-1}+r_{2 M-1}
\end{array}\right]}_{\boldsymbol{R}_{d}} \underbrace{\left[\begin{array}{c}
d_{M-1} \\
d_{M-2} \\
\vdots \\
d_{0}
\end{array}\right]}_{\boldsymbol{d}}=-\underbrace{\left[\begin{array}{c}
\left(r_{0}+r_{2 M}\right) \\
\left(r_{1}+r_{2 M+1}\right) \\
\vdots \\
\left(r_{M-1}+r_{3 M-1}\right)
\end{array}\right]}_{\boldsymbol{r}_{d}}
$$

由于矩阵 $\boldsymbol{R}_{\boldsymbol{d}}$ 是满秩的, 同样有

$$
\boldsymbol{d}=-\boldsymbol{R}_{d}^{-1} \boldsymbol{r}_{d}
$$

利用离散信号 $k$ 阶差分能量算子

$$
\gamma_{\kappa}[r(n)]=r(n) r(n+k-2)-r(n-1) r(n+k-1),
$$

和能量算子 [9]

$$
\psi_{d}[r(n)]=\gamma_{2}[r(n)]=[r(n)]^{2}-r(n-1) r(n+1),
$$

可求得差分方程的系数 $\boldsymbol{d}$ 为离散信号能量函数的函数(见附录 $\mathrm{A}$ )

$$
d_{i}=F_{4}\left(\gamma_{\kappa}[r(n-m)]\right), \quad i=0,1, \cdots, M-1,
$$

理论分析，可发现 $\boldsymbol{d}$ 中各元素为

$$
d_{0}=\sum_{\left(v_{1} \neq v_{2} \cdots \neq v_{M}\right)}(-1)^{M} v_{1} \cdot v_{2} \cdots v_{M}, \cdots, d_{M-2}=\sum_{\left(v_{1} \neq v_{2}\right)} v_{1} \cdot v_{2}, d_{M-1}=-\sum_{\left(v_{1}\right)} v_{i},
$$

其中

$$
v_{1}, v_{2}, \cdots, v_{M} \in\left\{\mathrm{e}^{-\mathrm{j} \Omega_{1}\left(n_{0}\right)}, \mathrm{e}^{-\mathrm{j} \Omega_{2}\left(n_{0}\right)}, \mathrm{e}^{-\mathrm{j} \Omega_{3}\left(n_{0}\right)}, \cdots, \mathrm{e}^{-\mathrm{j} \Omega_{M}\left(n_{0}\right)}, \mathrm{e}^{\mathrm{j} \Omega_{1}\left(n_{0}\right)}, \mathrm{e}^{\mathrm{j} \Omega_{2}\left(n_{0}\right)}, \mathrm{e}^{\mathrm{j} \Omega_{3}\left(n_{0}\right)}, \cdots, \mathrm{e}^{\mathrm{j} \Omega_{M}\left(n_{0}\right)}\right\},
$$

故 $\left\{\mathrm{e}^{-\mathrm{j} \Omega_{1}\left(n_{0}\right)}, \mathrm{e}^{-\mathrm{j} \Omega_{2}\left(n_{0}\right)}, \mathrm{e}^{-\mathrm{j} \Omega_{3}\left(n_{0}\right)}, \cdots, \mathrm{e}^{-\mathrm{j} \Omega_{M}\left(n_{0}\right)}, \mathrm{e}^{\mathrm{j} \Omega_{1}\left(n_{0}\right)}, \mathrm{e}^{\mathrm{j} \Omega_{2}\left(n_{0}\right)}, \mathrm{e}^{\mathrm{j} \Omega_{3}\left(n_{0}\right)}, \cdots, \mathrm{e}^{\mathrm{j} \Omega_{M}\left(n_{0}\right)}\right\}$ 为下式特征方程 的根:

$$
\sum_{i=0}^{M} d_{M-i} z^{-i}+\sum_{i=1}^{M} d_{i} z^{-M-i}=0,
$$

这样, 与连续信号一样, 可得离散信号各个信号分量的数字瞬时频率 $\Omega_{1}\left(n_{0}\right), \Omega_{2}\left(n_{0}\right)$, $\cdots, \Omega_{M}\left(n_{0}\right)$ 也为离散信号高阶差分能量函数的函数.

$$
\Omega_{i}\left(n_{0}\right)=F_{5}\left(F_{4}\left(\gamma_{\kappa}\left[r\left(n_{0}-m\right)\right]\right)\right), \quad i=1,2, \cdots, M .
$$

估计出信号分量的瞬时频率后, 即可利用瞬时频率和信号的能量函数以及高阶差分能量函数 进一步估计各个信号分量的瞬时幅度.

定义

$$
\psi_{d}\left[\Delta_{s}^{m} r, \Delta_{s}^{n} r\right]=\Delta_{s}^{m} r(n) \Delta_{s}^{n} r(n)-\Delta_{s}^{m} r(n-1) \Delta_{s}^{n} r(n+1),
$$

其中 $\Delta_{s} r=[r(n-1)-r(n+1)] / 2$, 并有 $\Delta_{s}^{m} r=\Delta_{s}\left(\Delta_{s}^{m-1} r\right), \psi_{d}\left[\Delta_{s}^{n} r\right]=\psi_{d}\left[\Delta_{s}^{n} r, \Delta_{s}^{n} r\right]$.

对于多个信号分量瞬时幅度有如下等式: 


$$
\begin{aligned}
& e_{0} \boldsymbol{\psi}_{d}[r]-e_{2} \boldsymbol{\psi}_{d}\left[\Delta_{s}{ }^{2} r\right]-e_{3}\left(\boldsymbol{\psi}_{d}\left[\Delta_{s}{ }^{4} r, \Delta_{s}{ }^{2} r\right]+\boldsymbol{\psi}_{d}\left[\Delta_{s}{ }^{2} r, \Delta_{s}{ }^{4} r\right]\right)-\cdots-e_{k} \sum_{i=1}^{k-1} \psi_{d}\left[\Delta_{s}{ }^{2 i} r, \Delta_{s}{ }^{2 k-2 i} r\right] \\
& -\cdots-e_{M-1} \cdot \sum_{i=1}^{(M-1)-1} \boldsymbol{\psi}_{d}\left[\Delta_{s}{ }^{2 i} r, \Delta_{s}{ }^{2(M-1)-2 i} r\right]-e_{M} \sum_{i=1}^{M-1} \psi_{d}\left[\Delta_{s}{ }^{2 i} r, \Delta_{s}{ }^{2 M-2 i} r\right] \\
= & e_{0} \sum_{i}^{M} a_{i}^{2}\left(n_{0}\right) \sin ^{2} \Omega_{i}\left(n_{0}\right)-2 e_{2} \sum_{i=1}^{M} a_{i}^{2}\left(n_{0}\right) \sin ^{6} \Omega_{i}\left(n_{0}\right)+2 e_{3} \sum_{i=1}^{M} a_{i}^{2}\left(n_{0}\right) \sin ^{8} \Omega_{i}\left(n_{0}\right) \\
& +\cdots+(-1)^{k-1}(k-1) c_{k} \sum_{i=1}^{M} a_{i}^{2}\left(n_{0}\right) \sin ^{2 k+2} \Omega_{i}\left(n_{0}\right)+\cdots+(-1)^{(M-1)-1}(M-2) e_{M-1} \\
& \cdot \sum_{i=1}^{M} a_{i}^{2}\left(n_{0}\right) \sin ^{2(M-1)+2} \Omega_{i}\left(n_{0}\right)+(-1)^{M-1}(M-1) e_{M} \sum_{i=1}^{M} a_{i}^{2}\left(n_{0}\right) \sin ^{2 M+2} \Omega_{i}\left(n_{0}\right),
\end{aligned}
$$

其中 $e_{M}=1, e_{i}(i=0,1, \cdots, M-1)$ 与连续信号的系数 $c_{i}(i=0,1, \cdots, M-1)$ 一样也是基本对称多项 式, 为

$$
\begin{aligned}
& e_{0}=2 \sin \Omega_{1}^{2} \cdot 2 \sin \Omega_{2}^{2} \cdots 2 \sin \Omega_{M}^{2}, \\
& \cdots \\
& e_{M-2}=\sum_{i, j=1,(i<j)}^{M} 2 \sin \Omega_{i}^{2} \cdot 2 \sin \Omega_{j}^{2}, \\
& e_{M-1}=\sum_{i=1}^{M} 2 \sin \Omega_{l}^{2} .
\end{aligned}
$$

错误! 未找到引用源。式同样可以进行扩展构成 $M$ 个方程. 求解该 $M$ 个方程可以得到各个信 号分量瞬时幅度 $a_{1}\left(n_{0}\right), a_{2}\left(n_{0}\right), \cdots, a_{M}\left(n_{0}\right)$ 的估计

$$
a_{i}\left(n_{0}\right)=F_{6}\left(\boldsymbol{\psi}_{c}\left[\Delta^{\boldsymbol{p}} r\right], \boldsymbol{\psi}_{c}\left[\Delta^{\boldsymbol{n}} r, \Delta^{\boldsymbol{m}} r\right], \boldsymbol{e}^{\prime}\right), \quad i=1, \cdots, M,
$$

式中 $\boldsymbol{n}_{l}=(2 l+k), \boldsymbol{m}_{l}=(2+k, 4+k, \cdots, 2 M-2 l+k), l=1,2, \cdots, M-1, k=0,1, \cdots, M-1$, 当 $M$ 为 奇数时 $\boldsymbol{p}=(0,1, \cdots, 2 M-2)$; 当 $M$ 为偶数时 $\boldsymbol{p}=(0,1, \cdots, 2 M-1), \boldsymbol{e}^{\prime}=\left(e_{0}, e_{1}, e_{3}, e_{4}, \cdots, e_{M}\right)$.

对 $n_{0}$ 一般化, 可以得到全部采样时刻的各个信号分量的瞬时频率和瞬时幅度估计值, 然 后重构各个信号分量, 即可实现对信号分量的分离.

\section{3 仿真以及性能分析}

假设接收的信号包含多个信号分量, 信号分量为幅度和频率慢变化的AM-FM信号. 在指 定仿真信号参数前先定义几个性能参数：归一化载波分离参数NCS(normalized carrier separation)、平均功率比MPR(mean power ratio)、载频带宽比 CR/IB(carrier to information bandwidth ratio)、载频频偏比 $\mathrm{CR} / \mathrm{FD}$ (carrier to frequency deviation ratio) $)^{[10]}$. 其定义表达式如下:

$$
\begin{gathered}
\operatorname{NCS}=\left|\Omega_{c 2}-\Omega_{c 1}\right| / \sum_{i}\left(k_{f_{i}}+\Omega_{a i}+\Omega_{f i}\right), \\
\operatorname{MPR}(\mathrm{dB})=20 \log \left(\sigma_{x_{i}} / \sigma_{x_{j}}\right), i \neq j,
\end{gathered}
$$




$$
\begin{gathered}
(\mathrm{CR} / \mathrm{IB})_{\mathrm{i}}=\Omega_{c i} / \max \left(\Omega_{f i}, \Omega_{a i}\right), \\
(\mathrm{CR} / \mathrm{FD})_{\mathrm{i}}=\Omega_{c i} / k_{f_{i}},
\end{gathered}
$$

其中 $\Omega_{c i}, \Omega_{f i}, \Omega_{a i}$ 分别为信号分量的数字载频、频率调制信号的最高数字频率、幅度调制信 号的最高数字频率; $k_{f i}$ 为频率调制系数.

NCS 用来测量信号分量的频谱分离特性, NCS 越小表明信号分量的频谱重叠越严重; MPR 用来测量信号分量的功率比, MPR 太大或太小表明信号分量的功率差别较大; CR/IB 用来测量 信号的调制变化相对于载波的快慢, $\mathrm{CR} / \mathrm{IB}$ 越小表明信号分量调制变化相对于载频越快; $\mathrm{CR} / \mathrm{FD}$ 用来测量频率调制的强度, $\mathrm{CR} / \mathrm{FD}$ 越小表明信号分量频率调制越大.

仿真假定接收信号包含 5 个时频重叠的信号分量, 其幅度、频率调制信号利用AR模型产 生. 仿真产生的信号参数以及定义的各个性能参数的值如 表 1 .

\section{表 1 仿真信号的参数设置}

\begin{tabular}{cccccc}
\hline 信号参数 & 取值 $/ \mathrm{KHz}$ & 信号参数 & 取值 $/ \mathrm{KHz}$ & 信号参数 & 取值 \\
\hline$f_{s}$ & 10 & $f_{c_{2}}=f_{s} / 8+f_{s} / 100$ & 1.35 & $\mathrm{NCS}$ & 0.2427 \\
$k_{a_{i}}$ & 1 & $f_{c_{1}}=f_{s} / 8-f_{s} / 100$ & 1.15 & $\mathrm{MPR}$ & $0 \mathrm{~dB}$ \\
$\theta_{i}$ & 0 & $f_{c_{4}}=f_{s} / 8+2 f_{s} / 100$ & 1.45 & $(\mathrm{CR} / \mathrm{IB})_{\mathrm{i}}$ & $10.5,11.5,12.5,13.5,14.5$ \\
$f_{c_{3}}=f_{s} / 8$ & 1.25 & $f_{c_{5}}=f_{s} / 8-2 f_{s} / 100$ & 1.05 & $(\mathrm{CR} / \mathrm{FD})_{\mathrm{i}}$ & 200 \\
& & $f_{A M_{i}}, f_{F M_{i}}=f_{s} / 100$ & 0.10 & $k_{f_{i}}=f_{c_{i}} / 200$ & $5.25,5.75,6.25,6.75,7.25$ \\
\hline
\end{tabular}

表中 $f_{s}$ 为采样频率, $f_{c_{i}}$ 为各个信号分量的载频, $\theta_{i}$ 为各个信号分量的初始相位, $k_{f_{i}}$ 和 $k_{a_{i}}$ 为各个信号分量的频率调制系数和幅度调制系数, $f_{F M_{i}}$ 和 $f_{A M_{i}}$ 为各个信号分量的频率调 制信号和幅度调制信号的最高频率.

图 1 为接收到的包含 5 个信号分量信号的时、频域波形, 从中可以看出信号分量时域完全 重叠, 频域部分重叠, 重叠情形可见表 1 的参数 $\mathrm{NCS}=0.2427$, 重叠非常严重. 利用本文的离散 能量分量方法对 5 个信号分量的瞬时幅度和瞬时频率进行估计, 然后通过平滑和中值滤波. 估 计结果如图 2 和 3. 从图中可以看出估计值非常接近各个信号分量的真实值, 只是在信号分量
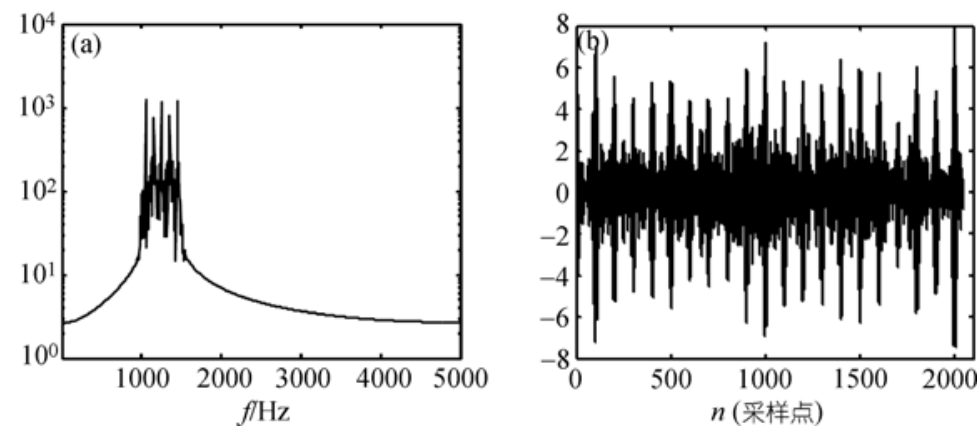

图 1 接收信号(包含 5 个信号分量)的时域和频域波形

(a) 接收信号频域波形; (b) 接收信号时域波形 

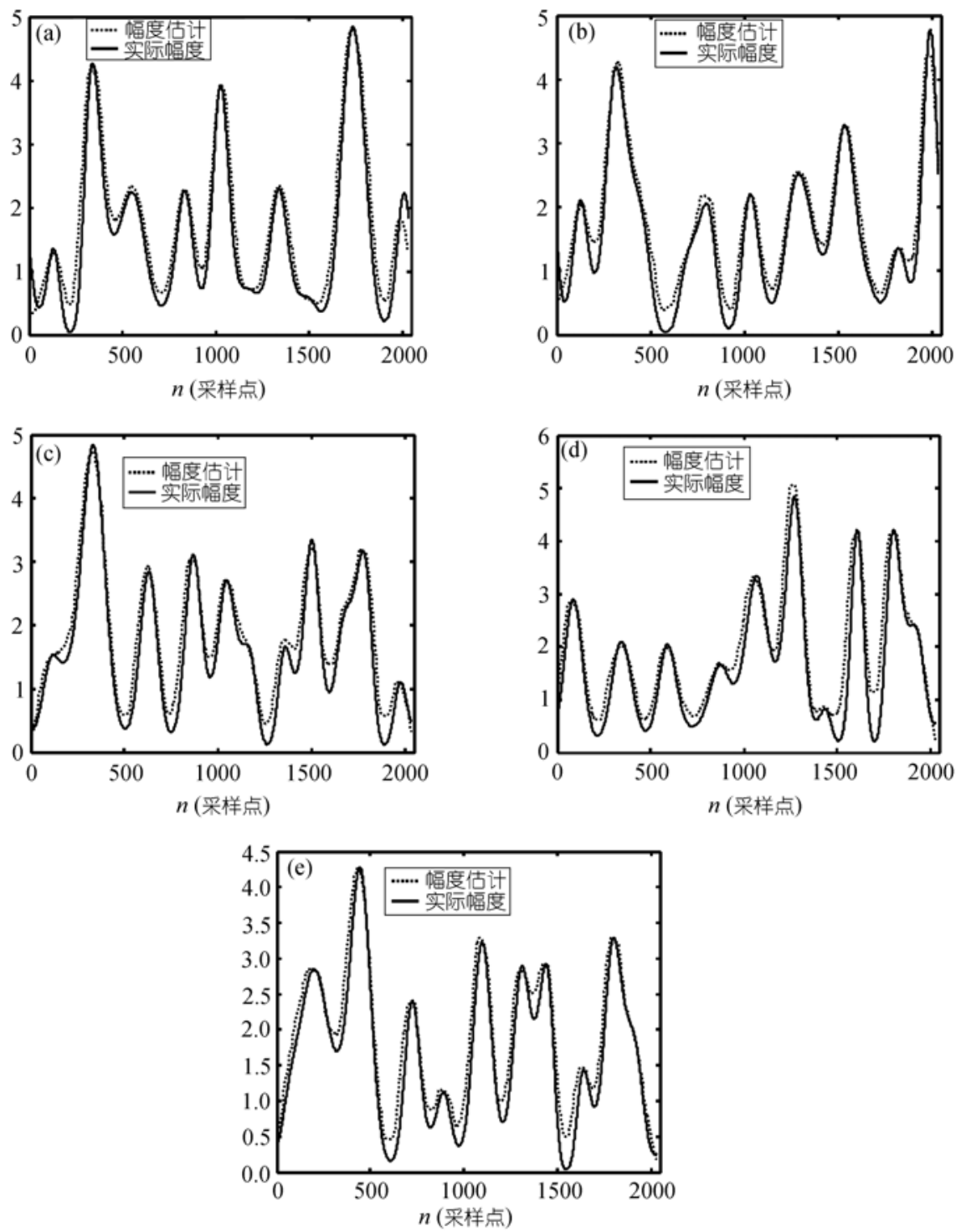

图 25 个信号分量的瞬时幅度估计

(a)第 1 个信号分量的幅度估计; (b)第 2 个信号分量的幅度估计; (c)第 3 个信号分量的幅度估计; (d)第 4 个信号分量的 幅度估计; (e)第 5 个信号分量的幅度估计

的瞬时频率和幅度的波峰和波谷这些变化较快的地方误差较大些，这是由于瞬时频率和幅度 变化较快时, 信号分量的单频假设会产生较大误差, 因而造成估计的瞬时频率和幅度产生较 大误差，这与前面的理论分析是一致的.

通过仿真试验以及分析还可以发现：NCS 减小, 信号分量的频谱重叠加重, 估计误差增大; MPR 太大或太小, 即信号分量的功率差别较大时, 大信号分量占据混和信号的能量的主要部 分, 其幅度估计误差较小, 而小信号分量的幅度估计误差则增大, 由于能量分离方法是由 
GDE(generating differential equation)推导出来的, 因而 MPR 的变化对于信号分量的频率估计 误差没有太大影响; CR/IB 减小, 即调制信号分量变化相对于载频加快, 估计误差增大; $\mathrm{CR} / \mathrm{FD}$ 减小, 即信号分量频率调制增大, 估计误差增大; 并且随着信号分量个数的增大, 信号分量的 瞬时幅度和瞬时频率的估计误差增大. 统计分析各种情形的估计误差如表 2.
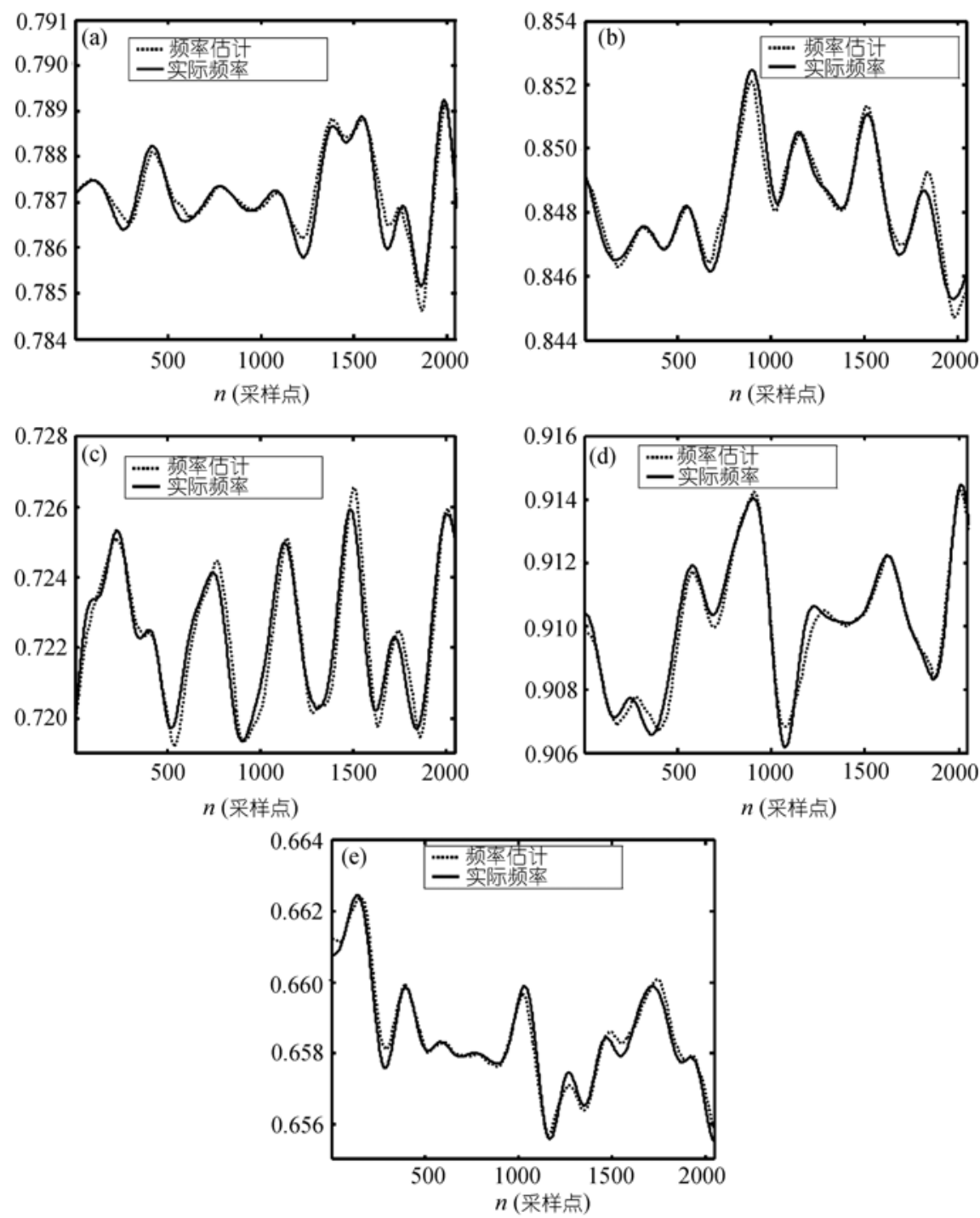

图 35 个信号分量的瞬时频率估计

(a)第 1 个信号分量的频率估计; (b)第 2 个信号分量的频率估计; (c)第 3 个信号分量的频率估计; (d)第 4 个信号分量的 频率估计; (e)第 5 个信号分量的频率估计

从表中可以看出随着信号分量个数的增加估计误差明显增大, 其原因是随着信号分量个 数的增加信号分量的频谱重叠增大, 表中对于 NCS 的定义是两个信号分量的定义, 如 
错误! 未找到引用源。式, 对于多个信号分量的计算是选取载频相邻的两个信号分量计算其 NCS, 因而其值不随信号分量的增加变化, 而事实上对于相同的调制信号参数以及相同的信 号分量载频间隔，随着信号分量的增加，信号分量的频域重叠明显增加.

表 2 多个信号分量的估计的平均绝对误差/\%

\begin{tabular}{|c|c|c|c|c|c|c|c|c|c|c|c|c|c|}
\hline \multirow{3}{*}{$\mathrm{CR} / \mathrm{IB}$} & \multirow{3}{*}{$\mathrm{CR} / \mathrm{FD}$} & \multicolumn{3}{|c|}{2 个信号 } & \multicolumn{3}{|c|}{3 个信号 } & \multicolumn{3}{|c|}{4 个信号 } & \multicolumn{3}{|c|}{5 个信号 } \\
\hline & & \multicolumn{3}{|c|}{ NCS } & \multicolumn{3}{|c|}{ NCS } & \multicolumn{3}{|c|}{ NCS } & \multicolumn{3}{|c|}{ NCS } \\
\hline & & 0.5 & 1 & 5 & 0.5 & 1 & 5 & 0.5 & 1 & 5 & 0.5 & 1 & 5 \\
\hline \multirow{3}{*}{10} & 200 & 0.576 & 0.314 & 0.245 & 1.884 & 1.132 & 0.984 & 3.543 & 2.871 & 1.757 & 9.541 & 8.745 & 7.138 \\
\hline & 300 & 0.428 & 0.274 & 0.223 & 1.554 & 1.025 & 0.832 & 3.225 & 2.346 & 1.432 & 9.151 & 8.357 & 6.985 \\
\hline & 400 & 0.302 & 0.216 & 0.202 & 1.319 & 0.854 & 0.744 & 2.912 & 1.856 & 1.148 & 8.954 & 7.854 & 6.774 \\
\hline \multirow{3}{*}{50} & 200 & 0.358 & 0.284 & 0.203 & 1.454 & 0.942 & 0.784 & 3.192 & 2.175 & 1.543 & 9.114 & 8.187 & 6.857 \\
\hline & 300 & 0.286 & 0.225 & 0.185 & 1.326 & 0.882 & 0.651 & 2.554 & 1.769 & 1.214 & 8.474 & 7.748 & 6.651 \\
\hline & 400 & 0.221 & 0.198 & 0.167 & 1.008 & 0.797 & 0.635 & 2.121 & 1.224 & 1.012 & 8.058 & 7.283 & 6.447 \\
\hline \multirow{3}{*}{100} & 200 & 0.303 & 0.211 & 0.178 & 1.125 & 0.876 & 0.698 & 2.257 & 1.487 & 1.168 & 8.375 & 7.594 & 6.549 \\
\hline & 300 & 0.289 & 0.196 & 0.164 & 0.986 & 0.808 & 0.621 & 1.985 & 1.301 & 1.025 & 8.156 & 7.335 & 6.498 \\
\hline & 400 & 0.249 & 0.174 & 0.152 & 0.904 & 0.754 & 0.614 & 1.798 & 1.106 & 0.987 & 7.964 & 7.173 & 6.397 \\
\hline
\end{tabular}

在没有噪声时, 利用上述方法对多个重叠的信号分量可以进行很好的分离, 在有噪声情 形时则可采用修正能量算子的方法 ${ }^{[11]}$.

\section{4 结论}

本文提出一种单信道多个时频重叠信号分量的能量分离方法, 利用能量函数和高阶差分 能量函数对信号分量的瞬时幅度和瞬时频率进行估计, 得到其连续信号和离散信号情形的瞬 时幅度和瞬时频率估计的解析表达式, 从而对多个信号分量进行分离. 该方法计算简单, 具有 很好的时域分辨率以及瞬时自适应性, 能够对多个时频重叠信号分量的瞬时频率和幅度进行 准确的估计, 并且不需要知道信号的载频等先验信息, 因而具有很好的实时性和实用价值.

\section{参考文献}

1 Li T, Sidiropoulos N D. Blind digital signal separation using successive interference cancellation iterative least squares. IEEE Trans Signal Process, 2000, 48(11): 3146-3152 [DOI]

2 Peleg S, Friedlander B. Multicomponent signal analysis using polynomial phase transform. IEEE Trans Aerosp Eletron Syst, 1996, 32(1): 378-384 [DOI]

3 Barbarossa S, Scaglione A, Giannakis G B. Product high-order ambiguity function for multicomponent polynomial-phase signal modeling. IEEE Trans Signal Process, 1998, 46(3): 691-708 [DOD]

4 Barbarossa S, Petrone V. Analysis of polynomial-phase signals by the integrated generalized ambiguity function. IEEE Trans Signal Process, 1997, 45(2): 316-327 [DOI]

5 Teager H. Some observations on oral air flow during phonation. IEEE Trans Signal Process, 1980, 28(5): 599$601 \underline{\text { DOI }}$

6 Kaiser J F. On a simple algorithm to calculate the energy of a signal. In: Proc ICASSP' 90. Piscataway: IEEE Operation Center, 1990. 381-384

7 Maragos P, Kaiser J F, Quatieri T F. Energy separation in signal modulations with application to speech analysis. IEEE Trans Signal Process, 1993, 41(10): 3024-3051 [DOI] 
8 Kumaresan R, Sadasiv A G, Ramalingam C S, et al. Instantaneous non-linear operators for tracking multicomponent signal parameters. In: Proc SSAP’ 92. Piscataway: IEEE Operation Center, 1992. 404-407

9 Maragos P, Potamianos A. Higher order differential energy operators. IEEE Signal Process Lett, 1995, 2(8): $152-154 \underline{[\mathrm{DOI}]}$

10 Santhanam B, Maragos P. Multicomponent AM-FM demodulation via periodicity based algebraic separation and energy-based demodulation. IEEE Trans Commun, 2000, 48(3): 473-490 [DOI]

11 蔡权伟, 魏平, 肖先赐. 单信道多信号分量分离. 通信学报, 2006, 27(6): 49-56

\section{附录 A}

含有 $M$ 个信号分量的连续信号 $r(t)$ 的微分方程经过变换后的能量函数形式表达式为

$$
\left[\begin{array}{cccc}
\gamma_{2 M-1}\left[r^{M-1}\right] & \gamma_{2 M-3}\left[r^{M-1}\right] & \cdots & \gamma_{3}\left[r^{M-1}\right] \\
\gamma_{2 M-1}\left[r^{M-2}\right] & \gamma_{2 M-3}\left[r^{M-2}\right] & \cdots & \gamma_{3}\left[r^{M-2}\right] \\
\vdots & \vdots & & \vdots \\
\gamma_{2 M-1}[r] & \gamma_{2 M-3}[r] & \cdots & \gamma_{3}[r]
\end{array}\right]\left[\begin{array}{c}
c_{M-1} \\
c_{M-2} \\
\vdots \\
c_{1}
\end{array}\right]=-\left[\begin{array}{c}
\gamma_{2 M+1}\left[r^{M-1}\right] \\
\gamma_{2 M+1}\left[r^{M-2}\right] \\
\vdots \\
\gamma_{2 M+1}[r]
\end{array}\right],
$$

方程系数 $c_{0}$ 的能量函数形式表达式为

$$
\gamma_{2 M-3}[\ddot{r}] c_{M-1}+\cdots+\gamma_{3}[\ddot{r}] c_{2}-\gamma_{3}[r] c_{0}=-\gamma_{2 M-1}[\ddot{r}] \text {. }
$$

这样通过对 $c_{i}(i=0,1, \cdots, M-1)$ 关于信号能量函数的方程求解能够得到

$$
c_{i}=F_{1}\left(\gamma_{\kappa}\left[r^{(m)}\right]\right), \quad i=0,1, \cdots, M-1,
$$

式中 $F_{1}(\cdot)$ 表示变换函数. 各个系数 $c_{i}(i=0,1, \cdots, M-1)$ 的函数包含的高阶差分能量函数 $\gamma_{\boldsymbol{\kappa}}\left[r^{(\boldsymbol{m})}\right]$ 的阶数 $\boldsymbol{K}$ 和信号微分的阶数 $\boldsymbol{m}$ 的取值对于信号分量个数 $M \geqslant 3$ 的情形有:

当 $i=0$ 时,

$$
\boldsymbol{k}_{l}=(3), \boldsymbol{m}_{l}=(0,1 \cdots, M), l=1 ; \quad \boldsymbol{k}_{l}=(5,7 \cdots, 2 M-3,2 M-1), \quad \boldsymbol{m}_{l}=(2,3, \cdots, M), \quad l=2,3, \cdots, M ;
$$

当 $i=1, \cdots, M-1$ 时;

$$
\boldsymbol{k}_{l}=(2 l+1), \boldsymbol{m}_{l}=(0,1, \cdots, M-3, M-2), l=1,2, \cdots, M .
$$

当信号分量个数 $M=2$ 时微分方程系数为

$$
\begin{aligned}
& c_{0}=\gamma_{3}[\ddot{r}] / \gamma_{3}[r], \\
& c_{1}=\gamma_{5}[r] / \gamma_{3}[r] .
\end{aligned}
$$

含有 $M$ 个信号分量的离散信号 $r(n)$ 的差分方程经过变换后的能量函数形式表达式为

$$
\begin{aligned}
& {\left[\begin{array}{cccc}
\gamma_{M}\left[r_{2 M-1}\right]-\gamma_{M}\left[r_{M}\right] & \gamma_{M-1}\left[r_{2 M-2}\right]-\gamma_{M-1}\left[r_{M}\right] & \cdots & \boldsymbol{\psi}_{d}\left[r_{M+1}\right]-\psi_{d}\left[r_{M}\right] \\
\gamma_{M}\left[r_{2 M-2}\right]-\gamma_{M}\left[r_{M-1}\right] & \gamma_{M-1}\left[r_{2 M-3}\right]-\gamma_{M-1}\left[r_{M-1}\right] & \cdots & \boldsymbol{\psi}_{d}\left[r_{M}\right]-\boldsymbol{\psi}_{d}\left[r_{M-1}\right] \\
\vdots & \vdots & & \vdots \\
\gamma_{M}\left[r_{M}\right]-\gamma_{M}\left[r_{1}\right] & \gamma_{M-1}\left[r_{M-1}\right]-\gamma_{M-1}\left[r_{1}\right] & \cdots & \boldsymbol{\psi}_{d}\left[r_{2}\right]-\boldsymbol{\psi}_{d}\left[r_{1}\right]
\end{array}\right]\left[\begin{array}{c}
d_{M-1} \\
d_{M-2} \\
\vdots \\
d_{1}
\end{array}\right] } \\
= & -\left[\begin{array}{c}
\gamma_{M+1}\left[r_{2 M}\right]-\gamma_{M+1}\left[r_{M}\right] \\
\gamma_{M+1}\left[r_{2 M-1}\right]-\gamma_{M+1}\left[r_{M-1}\right] \\
\vdots \\
\gamma_{M+1}\left[r_{M+1}\right]-\gamma_{M+1}\left[r_{1}\right]
\end{array}\right] .
\end{aligned}
$$

方程系数 $d_{0}$ 的能量函数形式表达式为 


$$
\begin{aligned}
& d_{M-1}\left(\gamma_{M-1}\left[r_{2 M-1}\right]+\gamma_{M+1}\left[r_{2 M-1}\right]-\gamma_{M+1}\left[r_{M+1}\right]-\gamma_{M-1}\left[r_{M-1}\right]\right)+\cdots \\
& +d_{2}\left(\psi_{d}\left[r_{M+2}\right]+\gamma_{4}\left[r_{M+2}\right]-\gamma_{4}\left[r_{M+1}\right]-\boldsymbol{\psi}_{d}\left[r_{M-1}\right]\right) \\
& +d_{0}\left(\boldsymbol{\psi}_{d}\left[r_{M}\right]-\boldsymbol{\psi}_{d}\left[r_{M+2}\right]\right) \\
& =-\left(\gamma_{M}\left[r_{2 M}\right]+\gamma_{M+2}\left[r_{2 M}\right]-\gamma_{M+2}\left[r_{M+1}\right]-\gamma_{M}\left[r_{M-1}\right]\right) .
\end{aligned}
$$

通过对 $d_{i}(i=0,1, \cdots, M-1)$ 关于离散信号能量函数的方程求解能够得到

$$
d_{i}=F_{4}\left(\gamma_{\kappa}\left[r_{m}\right]\right), i=0,1, \cdots, M-1,
$$

式中 $F_{4}(\cdot)$ 表示变换函数, 其中各个系数 $d_{i}(i=0,1, \cdots, M-1)$ 的函数包含的离散信号高阶差分能量函数 $\gamma_{\boldsymbol{K}}\left[r_{\boldsymbol{m}}\right]$ 的阶数 $\boldsymbol{K}$ 和信号差分的阶数 $\boldsymbol{m}$ 的取值对于信号分量个数 $M \geqslant 3$ 的情形有:

当 $i=0$ 时,

$$
\begin{aligned}
& k_{l}=(l+1), \boldsymbol{m}_{l}=(M-1, M, \cdots, 2 M+1), l=1 ; \\
& k_{l}=(l+1), \boldsymbol{m}_{l}=(M-1, M, \cdots, 2 M+l), l=2,3, \cdots, M-1 ; \\
& k_{l}=(l+1), \boldsymbol{m}_{l}=(M-1, M, \cdots, 2 M+l-2), \quad l=M, M+1 .
\end{aligned}
$$

当 $i=1, \cdots, M-1$ 时,

$$
\begin{aligned}
& k_{l}=(M+2-l), \boldsymbol{m}_{l}=(M, M+1, \cdots, 2 M-2,2 M, 2 M+1, \cdots 3 M-1-l), l=1 ; \\
& k_{l}=(M+2-l), \boldsymbol{m}_{l}=(M, M+1, \cdots, 3 M-1-l), l=2,3, \cdots, M .
\end{aligned}
$$

当信号分量个数 $M=2$ 时差分方程系数为

$$
\begin{gathered}
d_{0}=\frac{\psi_{d}(r)-\psi_{d}\left(r_{3}\right)+\gamma_{4}\left(r_{2}\right)-\gamma_{4}\left(r_{3}\right)}{\psi_{d}\left(r_{1}\right)-\psi_{d}\left(r_{2}\right)}, \\
d_{1}=\frac{\gamma_{3}\left(r_{3}\right)-\gamma_{3}\left(r_{1}\right)}{\psi_{d}\left(r_{1}\right)-\psi_{d}\left(r_{2}\right)} .
\end{gathered}
$$

